\title{
The need for mechanical ventilation in a child exposed to a laundry detergent pod
}

\author{
Fulya Kamit-Can ${ }^{1}$, Caner Alparslan², Ayşe Berna Anı1 ${ }^{3}$, Murat Anı1 ${ }^{4}$, Neslihan Zengin ${ }^{1}$, \\ Ender Can ${ }^{1}$ \\ ${ }^{1}$ Pediatric Intensive Care Unit, ${ }^{2}$ Department of Pediatric Nephrology, and ${ }^{4}$ Pediatric Emergency Care Unit, Tepecik Training \\ and Research Hospital, ${ }^{3}$ Intensive Care Unit, Department of Pediatrics, Katip Çelebi Univercity, Faculty of Medicine, \\ Izmir, Turkey \\ E-mail: fulyakamit@yahoo.co.uk
}

Received: 28 July 2015, Revised: 12 April 2016, Accepted: 28 April 2016

\begin{abstract}
SUMMARY: Kamit-Can F, Alparslan C, Anıl AB, Anıl M, Zengin N, Can E. The need for mechanical ventilation in a child exposed to a laundry detergent pod. Turk J Pediatr 2016; 58: 323-326.
\end{abstract}

Laundry detergent pods (LDPs) are a new, concentrated form of detergent covered by a membrane of polyvinyl alcohol or other water-soluble material. In contrast to traditional laundry detergents, the spectrum of responses to exposure to LDPs ranges from mild to life-threatening events. This is a case report of a 3-year-old male who ingested part of an LDP, leading to a depressed level of consciousness, upper airway obstruction, and severe respiratory distress. The patient required intubation and mechanical ventilation for 2 days before being discharged. This rare, severe clinical pattern demonstrates the potential toxicity of these laundry detergents. In the literature, few cases that required intubation and ventilation have been reported. To our knowledge, this is also the first case of LDP exposure reported from Turkey.

Key words: laundry detergent pod, critically ill children, mechanical ventilation, poisoning.

Laundry detergent pods (LDPs) are a new form of concentrated laundry detergent covered by a membrane designed to dissolve in water, made from a material such as polyvinyl alcohol1-5. Most of these capsules are colorful and perceived as toys or candies by pediatric patients, especially those under 5 years of age ${ }^{2,6}$. This new detergent form has been marketed since the 2000s in Europe, and since 2011 in both the United States and Turkey. Unlike traditional laundry detergents, LDPs have been associated with some morbidity after pediatric exposure, although the exact spectrum of illnesses has not been established ${ }^{1,2,5}$. The spectrum of responses to exposure has ranged from mild to serious and life-threatening events. Conjunctivitis and keratitis are the most frequently seen pathologies after topical exposure. With systemic exposure, vomiting, swallowing difficulties, respiratory distress, and central nervous system depression may be seen ${ }^{2}$.

In this report, we share our experience with unusual features of LDP intoxication in a 3 -year-old male who experienced respiratory distress and nervous system depression that required 2 days of mechanical ventilation. To our knowledge, this is the first pediatric LDP intoxication report from Turkey, and it highlights the potentially significant toxicity of these common household cleaning agents.

\section{Case Report}

A previously healthy 3-year-old male presented to our emergency department (ED) after the accidental ingestion of an LDP (Ariel liquid gel capsule ${ }^{\circledR}$ ). His mother had found him with a half-bitten pod in his hands and green staining around his mouth and shirt. Within $10 \mathrm{~min}$ he developed profuse vomiting. After that, his mother brought him by car to our pediatric ED, which took a further $30 \mathrm{~min}$. Upon arrival, he showed drowsiness, tachypnea, inspiratory stridor, sternal and intercostal retraction, and difficulty swallowing. Initial vital signs were as follows: pulse rate 155/ 


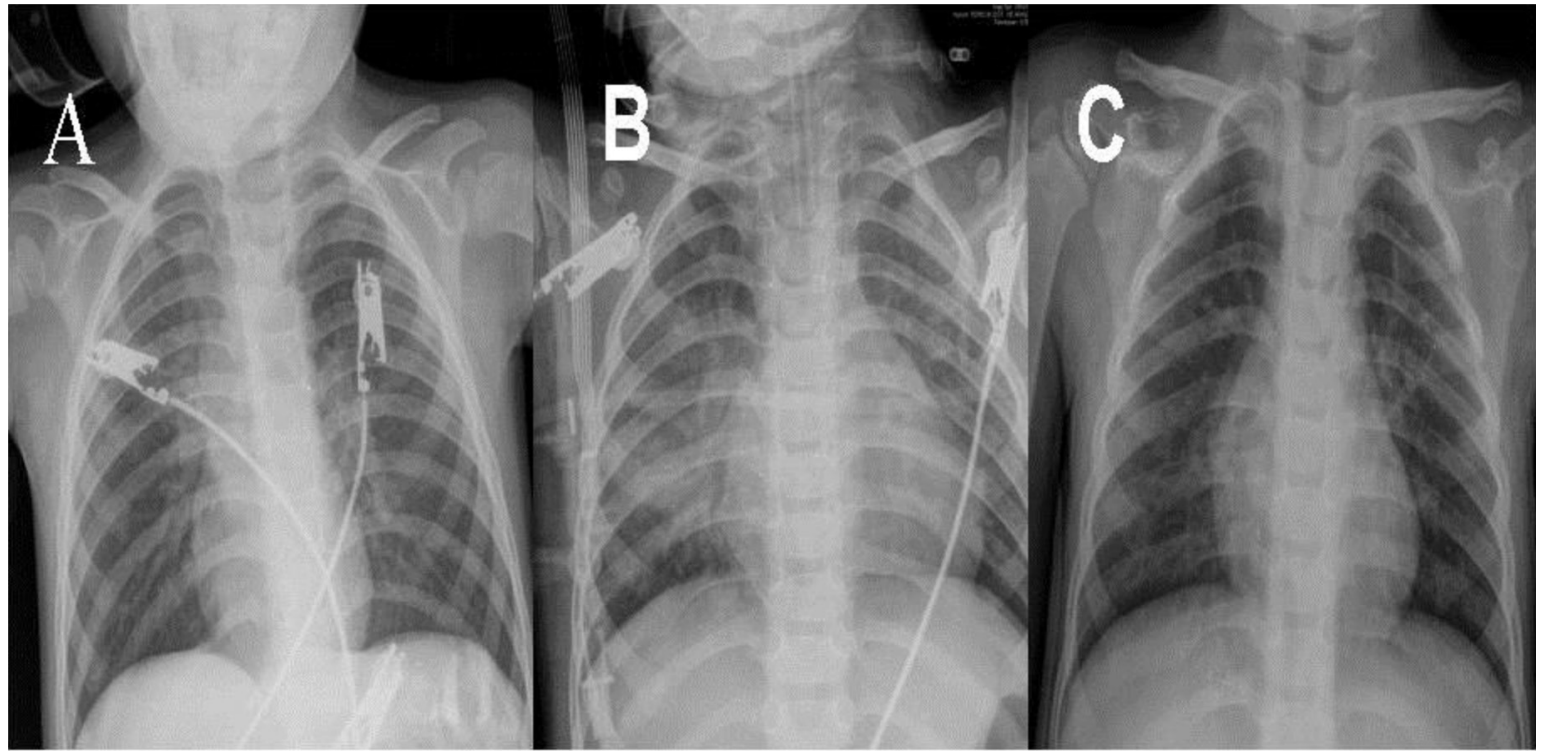

Fig. 1. Chest X-ray was normal at admission (A), infiltration on right middle zone in post-intubation radiography (B), normal radiological findings in post-extubation (C).

min; respiratory rate $30 / \mathrm{min}$; blood pressure 112/69 mmHg; capillary re-filling time $<2$ sec; oxygen saturation $94 \%$ (on oxygen); and a Glasgow Coma Scale (GCS) score of 13. On physical examination, coarse crackles were found bilaterally at pulmonary auscultation; the rest of the examination was unremarkable. During the emergency department observation, his respiratory signs deteriorated rapidly, the need for supplemental oxygen increased, and the patient was transferred to the pediatric intensive care unit (PICU). In laboratory analysis, his complete blood cell count, blood chemistry, troponin-I, serum C-reactive protein (CRP), and coagulation parameters were within normal limits. Venous blood gas analysis revealed a $\mathrm{pH}$ of $7.24 ; \mathrm{pCO}_{2}$ at $45.6 \mathrm{~mm} \mathrm{Hg} ; \mathrm{pO}_{2}$ at $63.4 \mathrm{~mm} \mathrm{Hg} ; \mathrm{HCO}_{3}$ at $18.2 \mathrm{mEq} / \mathrm{L}$; base excess of $-7.6 \mathrm{mEq} / \mathrm{L}$; and lactate level of $1.7 \mathrm{mmol} / \mathrm{L}$. A chest X-ray was unremarkable (Fig. 1A). Treatment with systemic steroids (dexamethasone 0.15 $\mathrm{mg} / \mathrm{kg} / \mathrm{dose}$ ) and adrenaline (3 $\mathrm{mg} /$ dose via nebulization) was started immediately for upper airway edema. Over a short period, stridor and bronchospasm progressed to compromise the patient's breathing, and his GCS score dropped rapidly to 11 . Because of the history of caustic agent ingestion and the rapidly developing stridor, the patient was determined to have a difficult airway. Therefore, a tracheostomy was performed and the patient was intubated with a
4-F uncuffed endotracheal tube $2 \mathrm{~h}$ after PICU admission. A mechanical ventilator was set to a pressure controlled-synchronized intermittent mandatory ventilation mode, with pressure control at $16 \mathrm{~cm} \mathrm{H}_{2} \mathrm{O}$, a post-expiratory end pressure of $5 \mathrm{~cm} \mathrm{H}_{2} \mathrm{O}$, a frequency of 25/ min, and a fraction of inspired oxygen $\left(\mathrm{FiO}_{2}\right)$ of $50 \%$. Empiric antibiotic treatment was started (ampicillin-sulbactam $100 \mathrm{mg} / \mathrm{kg} /$ day) and systemic steroids were continued. A postintubation chest X-ray showed infiltration in the right lung (Fig. 1B). During the invasive mechanical ventilation, bilateral rhonchi, a prolonged expiratory phase, and bronchospasm increased; blood-tinged aspirate was noted with suction. A beta agonist (salbutamol $0.15 \mathrm{mg} /$ $\mathrm{kg} /$ dose at 2-h intervals) was administered via jet nebulizer for bronchospasm. Ventilator adjustments were made to prolong expiratory time. The patient required $40 \%$ inspired oxygen and was weaned early. Resolution of the upper airway edema (leakage around the cuff) and bronchoconstriction was achieved. Mechanical ventilation had been maintained for 2 days before the patient was extubated. The post-extubation chest X-ray was unremarkable (Fig. 1C), and infiltration noted in the right lung was considered to be atelectasis. Blood gas analyses revealed a $\mathrm{pH}$ of $7.39, \mathrm{pCO}_{2}$ at $34 \mathrm{~mm} \mathrm{Hg}, \mathrm{pO}_{2}$ at $99 \mathrm{~mm} \mathrm{Hg}, \mathrm{HCO}_{3}$ at $23 \mathrm{mEq} / \mathrm{L}$, base excess of -1.2 , and a lactate level of $0.2 \mathrm{mmol} / \mathrm{L}$. After completion of 
the post-extubation period, the patient was transferred to the general pediatric ward and was discharged to home on hospital day 6 . He had no complaints of either swallowing or breathing difficulties at the end of the first week or at the 1-month follow up.

\section{Discussion}

This case represents a rare, severe clinical pattern after exposure to a laundry detergent pod. Progression to life-threatening conditions was rapid and was unanticipated after exposure to LDPs. This information should be kept in mind in cases such as ours, in which the patient shows rapidly deteriorating consciousness and severe respiratory distress, resulting in the need for invasive mechanical ventilation within hours.

In literature, the majority of similar cases are among children under 5 years of age. The most common route of exposure is determined ingestion, followed by eye and skin contact. Nearly all of the exposures were unintentional ${ }^{3,5,6}$, and may depend upon the attractiveness of LDPs, with their toy-like shape and colorful appearance ${ }^{6}$. Retrospective analyses of poison call-center enquiries from the US and the UK indicated that vomiting was by far the most common sign and symptom. With variable frequency the remaining symptoms included cough, eye irritation, nausea, and oral irritation ${ }^{3,5,6}$. In our patient, vomiting and cough were the initial symptoms.

Laundry detergent pod intoxication rarely causes catastrophic outcomes from effects on the nervous system. In the literature, there are few reports of central nervous system depression with respiratory distress, leading to intubation and mechanical ventilation ${ }^{1,2,5,6}$. In 2012, in a large case series of 647 patients from the UK, only two patients (both 2-yearold males) required mechanical ventilation. One of them had upper airway obstruction and the other had central nervous system depression ${ }^{5}$. In 2013, in a case series of patients with significant toxicity, three of four patients required mechanical ventilation. Two males, 17 and 20 months old, presented with respiratory distress and central nervous system depression. Their chest radiographs revealed infiltrative changes that disappeared later, consistent with atelectasis and similar to the changes observed in our patient. The third patient had upper airway injury, but the patient did not require mechanical ventilation. This patient also developed bronchospasm responsive to albuterol and methylprednisolone. The fourth patient, a 15-month-old female, was intubated for airway protection and hypoventilation ${ }^{1}$. In 2013, intubation and mechanical ventilation were reported in 2 of 187 patients 5 years old or younger who were exposed to laundry detergent pods ${ }^{6}$. Upper airway edema and pulmonary toxicity was seen, which unpredictably progressed to respiratory failure. Cough, wheezing, crackles, and retractions are the signs of respiratory system toxicityl,6. A 15-month-old female remained intubated for 5 days following laundry pod ingestion (Should this indicate reference 1 again, or is this a different patient than that noted earlier?). The use of inhaled bronchodilators for bronchospasm during mechanical ventilation has been reported in cases of pulmonary toxicity similar to ours ${ }^{2}$. In our case, rapid deterioration of the GCS score, distinct upper airway obstruction, and signs of pulmonary toxicity without oral cavity lesions led to intubation and mechanical ventilation. During the course of intensive care, severe bronchospasm responded to intensive bronchodilator treatment.

The potential mechanism(s) of toxicity after LDP exposure are unknown, and may differ according to the compounds present in LDPs. Other authors have indicated that ethanol, propylene glycol (metabolized to lactate), and surfactant components were the mandatory indicators of toxic effects ${ }^{1,2}$. In our patient, we were unable to obtain either the specific ethoxylated alcohol composition of the ingested product or a blood ethanol level. The blood lactate level was increased, at $1.7 \mathrm{mmol} / \mathrm{L}$ (normal range, $0.3-1.3 \mathrm{mmol} / \mathrm{L}$ ), and blood gas analysis revealed metabolic acidosis.

Laundry detergent pod exposure among children has a variable, but generally more severe, clinical spectrum than exposure to traditional detergents ${ }^{6}$. In addition, the lack of a worldwide toxicosurveillance program and of awareness of this clinical spectrum makes this new product more dangerous than it might seem. New exposure codes for these products and the development of a worldwide 
toxicosurveillance program will minimize their negative influence on child health ${ }^{7,8}$. To contribute to public health knowledge regarding LDP exposure, we have presented this first case report from our country. Such cases highlight a general lack of awareness of the risks of exposure to LDPs.

\section{Conclusions}

This is, to our knowledge, the first pediatric LDP intoxication report from Turkey, and highlights the potentially significant toxicity of laundry detergent pods. Rapid airway obstruction and mental status deterioration can develop after exposure to LDPs. This lifethreatening condition requires fast and careful airway management in a pediatric intensive care unit.

\section{REFERENCES}

1. Michael C, Beuhler MD, Gala PK, et al. Laundry detergent "pod" ingestions: a case series and discussion of recent literature. Pediatr Emerg Care 2013; 29: 743-747.

2. Schneir AB, Rentmeester L, Clark RF, et al. Toxicity following laundry detergent pod ingestion. Pediatr Emerg Care 2013; 29: 741-742.

3. Centers for Disease Control and Prevention (CDC) Health hazards associated with laundry detergent pods - United States, May-June 2012. MMWR. 2012; 61: 825-829.

4. Williams H, Moyns E, Bateman DN, et al. Hazard of household cleaning products: a study undertaken by the UK National Poisons Information Service. Clin Toxicol (Phila) 2012; 50: 770-775.

5. Williams $\mathrm{H}$, Bateman DN, Thomas $\mathrm{SH}$, et al. Exposure to liquid detergent capsules: a study undertaken by the UK National Poison Information Service. Clin Toxicol (Phila) 2012; 50: 776-780.

6. Forrester MB. Comparison of pediatric exposures to concentrated "pack" and traditional laundry detergents. Pediatr Emerg Care 2013; 29: 482-486.

7. Forrester MB. Surveillance detection of concentrated laundry detergent pack exposures. Clin Toxicol (Phila) 2012; 50: 847-850.

8. Scharman EJ. Liquid "laundry pods:" a missed global toxicosurveillance opportunity. Clin Toxicol(Phila) 2012; 50: 725-726. 